\title{
HIGH BRIGHTNESS INJECTORS BASED ON PHOTOCATHODE DC GUN*
}

\author{
Byung C. Yunn ${ }^{\dagger}$ \\ Thomas Jefferson National Accelerator Facility, Newport News, VA 23606, USA
}

\begin{abstract}
Sample results of new injector design method based on a photocathode dc gun are presented, based on other work analytically proving the validity of the emittance compensation scheme for the case even when beam bunching is involved. We have designed several new injectors appropriate for different bunch charge ranges accordingly. Excellent beam quality produced by these injectors clearly shows that a photocathode dc gun can compete with a rf gun on an equal footing as the source of an electron beam for the bunch charge ranging up to 2 nano Coulomb (nC). This work therefore elevates a dc gun based injector to the preferred choice for many ongoing high brightness accelerator projects considering the proven operational stability and high average power capability of the de gun.
\end{abstract}

\section{INTRODUCTION}

The very successful Jlab IR FEL[1] is based on a photocathode dc gun operating at $320 \mathrm{kV}$. The gun design started as a $500 \mathrm{kV}$ gun with a peak electric field of 10 $\mathrm{MV} / \mathrm{m}$ at the surface of the cathode. Due to breakdown problems the gun has been modified to a lower gradient ( 6 $\mathrm{MV} / \mathrm{m}$ ) with a spacer inserted. We are operating this gun at $320 \mathrm{kV}$ as mentioned. Despite technical difficulties to be overcome, Jlab is planning to develop a high voltage gun running as high as $750 \mathrm{kV}$. In the meantime, a $10 \mathrm{~kW}$ IR FEL upgrade under construction will most likely use the existing $320 \mathrm{kV}$ gun. A doubling of beam current from 5 to $10 \mathrm{~mA}$ and a higher beam energy of $200 \mathrm{MeV}$ will suffice for producing the $10 \mathrm{~kW}$ light intended. This necessitates an increase of the nominal bunch charge from the present $60 \mathrm{pC}$ to $135 \mathrm{pC}$. The expected beam quality with the presently installed injector in our best optimized setup is shown in Table 1. Values for emittances and bunch length are all for the rms quantities.

Table 1: Performance of the current IRFEL injector.

\begin{tabular}{|l|c|c|}
\hline Bunch Charge & $\mathbf{6 0} \mathbf{~ p C}$ & $\mathbf{1 3 5} \mathbf{~ p C}$ \\
\hline$\varepsilon_{\mathrm{x}}(\mathrm{mm}-\mathrm{mrad})$ & 5 & 10 \\
\hline$\varepsilon_{\mathrm{z}}(\mathrm{deg}-\mathrm{keV})$ & 10 & 14 \\
\hline$\sigma_{\mathrm{E}} / \sigma_{\mathrm{z}}(\mathrm{keV} / \mathrm{ps})$ & $12 / 1.4$ & $21 / 1.5$ \\
\hline
\end{tabular}

Although this performance of the IR FEL injector meets the beam quality specifications set for the upgrade with a

\footnotetext{
*Work supported by the U. S. Department of Energy under contract DEAC05-84-ER40150, the Office of Naval Research, the Commonwealth of Virginia, and the Laser Processing Consortium.

†yunn@jlab.org
}

sufficient margin, it became clear that an improved design must be found before a photocathode dc gun-based injector can be competitive as the electron beam source for much more demanding accelerators under study at the Jefferson Lab and other places. Such efforts include energy recovering linac (ERL)-based light source projects like Cornell ERL, JERBIL, or future Jlab FEL upgrades to a UV spectral range. It is in this context that we initiated a search for an alternative injector design which does a significantly better job in preserving a good beam quality started at the dc gun[2].

\section{DESIGN APPROACHES}

Electron bunches produced at a dc gun tend to be very long due to a low beam energy of only a few hundreds $\mathrm{keV}$. In a typical thermionic gun the beam is continuous while the beam is bunched in a typical photocathode dc gun with the bunch length in the range of about 100 pico seconds (ps). As a result, the bunch needs to be bunched further at least by an order of magnitude (in our present case) at some point in the injector before the beam is delivered to the main linac. Chopping can provide a shorter bunch but is to be avoided to maximize beam power. In most situations chopping is not necessary with this type of gun. For a high charged bunch, roughly from 10 pC upward, space charge force effects must be included in the calculation of injector beam dynamics. The beam quality can degrade rather quickly and the bunch shape changes substantially during the beam transportation. Therefore, it is a necessary rule that the acceleration of the bunch should take place at the earliest possible opportunity in order to minimize the distance the beam is transported at low energy.

One often finds that the emittance at the gun exit already has grown so much that little room is left for an additional increase in a subsequent beam line, which inevitably happens even in an ideal machine where one could accelerate the beam right out of the gun. Fortunately, there is an extremely useful physical mechanism which makes an elimination of a large portion of space chargeinduced emittance degradations possible, known as the emittance compensation scheme[3]. It has been applied successfully to the design of a photocathode rf gun-based injector. However, the beam characteristics from an rf gun is quite different from that of a dc gun. First, the beam energy at the gun exit is typically an order of magnitude higher, and secondly, the bunch is short enough that no further bunching is necessary.

There are two obvious approaches in designing an injector based on a photo-cathode dc gun. One is first to 
accelerate the beam to a higher energy, say to 5 to 10 $\mathrm{MeV}$, and then to bunch in a magnetic chicane because a velocity bunching is not effective at such energies. This is the preferred approach if one is going to apply the conventional emittance compensation scheme to minimize any emittance degradation induced by space charge force. We have studied this option to design an injector for a light source project based on the ERL technology pioneered at the Jefferson Lab. See ref. [4] for more details. However, we found that an injector design following this path is confronted with a difficult problem of dealing with a very large longitudinal phase space as a result of a long bunch coming out of a dc gun. One must show that the longitudinal emittance can be reduced to a desired level while maintaining small transverse emittances in the process. It won't be easy because of a large energy spread present in the beam at a subsequent beam transport system which necessarily involves many bends, even though space charge effects play a lesser role there.

The other approach is to bunch the beam first when the beam is still soft and then to accelerate to a final energy. This is the path we took in designing our current FEL injector (and CEBAF main injector for that matter). This, of course, has resulted in an injector whose best performance is shown in Table 1. A detailed study on the development of transverse emittances along the injector line components has shown that there are no emittance compensations. From this one could conclude that the bunching process destroys the emittance compensation mechanism. However, with a closer look at the problem, we were able to prove that the emittance compensation scheme should work equally well when a bunching process is linear[5]. Indeed we find the condition for a perfect cancellation:

$z=\left(f+z_{1}-\alpha^{-1}\right) \log \left(1-\alpha z_{1}-\alpha z\right)-\left(z_{1}-\alpha^{-1}\right) \log \left(1-\alpha z_{1}\right)$

which reduces to the Carsten's relation:

$z^{2}=2 f\left(z+z_{1}\right)$

with no bunching at all. Here $\alpha$ is the bunching rate and a thin lens with the focal length $f$ is located at $z_{1}$. As in the original case the emittance is completely restored at $z$ downstream of the lens.

Armed with this new finding we have designed several new injectors (one each for different bunch charge range), which we briefly describe in section 3 . The final achieved emittances show that a substantial portion of space charge-induced increases has been actually removed. As a result, we are able to deliver an electron beam whose emittance is even lower than the value it has achieved at the exit of the gun. Remarkably this has been accomplished while the bunch length has been shortened at least by a factor of 10 in the process. The evaluation of designs has been carried out with PARMELA[6] code provided by LANL.

\section{NEW INJECTORS}

We should start by mentioning that we are not reporting on the design of an injector for any particular project in this paper. It is simply a general study to understand features of an injector based on a dc gun.

For all designs we present here we assume a Gaussian bunch with the rms bunch length of 20 ps longitudinally cutoff at $+/-2 \sigma_{z}$. Transversely the laser spot is round and the distribution is approximately Gaussian. Injector layout is deceptively simple. It consists of a CEBAF-type dc gun which we assume to be operating at $500 \mathrm{kV}$, a buncher operating at $1497 \mathrm{MHz}$, and a CEBAF cryounit which consists of two 5-cell superconducting CEBAF cavity with the fundamental mode at $1497 \mathrm{MHz}$ separated by a quarter meter. The cavity gradient required is $10 \mathrm{MV} / \mathrm{m}$ which is quite modest in today's SRF technology standard.

\subsection{Low Bunch Charge: Less than $100 \mathrm{pC}$}

The ERL technology appears to be gaining popularity in these days. The technology offers a clear advantage in building a high power $\mathrm{cw}$ machine. Requirement on $\mathrm{rf}$ power consumption and on beam dump capacity is drastically reduced. There are several projects under intensive study employing the technology to build a high average current electron linac (100 $\mathrm{mA}$ and above) for various purposes. One of them is the Cornell ERL light source project, which we consider to be the representative case for any similar high brightness and high power light source project. We will discuss the required beam quality and present our injector performance. High beam quality is demanded by the project, as can be seen from the Cornell ERL design report[7]. It is required for the injector:

- to deliver a $77 \mathrm{pC}$ electron bunch at $1.3 \mathrm{GHz}$ repetition rate producing an average beam current of $100 \mathrm{~mA}$ and

- to deliver a very low transverse emittance beam on the order of $2 \mathrm{~mm}$-mrad. The rms bunch length is required to be as short as $2.3 \mathrm{ps}$ at the end of injector.

Following the second approach advocated in the previous Section we have found an injector solution which satisfies the above injector requirements. Our injector is built on the CEBAF cavity which operates at $1497 \mathrm{MHz}$. The bunch charge is chosen to be $80 \mathrm{pC}$. Therefore, the injector will be capable of delivering the average current upto $120 \mathrm{~mA}$. The beam quality achieved at the end of injector is listed in Table 2. One should compare the longitudinal emittance with that of other designs based on the first approach described in the Section 2 to appreciate[4].

Finally, we would like to note an interesting feature of our injector. Even though our nominal gun voltage is assumed to be $500 \mathrm{kV}$, we find that the injector could tolerate a lower gun voltage down to $400 \mathrm{kV}$ resulting in 
only a slight degradation to beam quality. For instance, we obtain $\varepsilon_{\mathrm{x}}{ }^{\text {rms-normalized }}=1.0 \mathrm{~mm}$-mrad and $\varepsilon_{\mathrm{z}}{ }^{\text {rms-normalized }}$ $=6.0$ deg-keV if we had a $400 \mathrm{kV}$ gun. This feature may turn out to be of a real practical value when operating the injector, especially because of the problems we faced as mentioned in the Introduction in connection with the operational experience of the Jlab IR FEL Demo gun.

Table 2: Beam quality for an $80 \mathrm{pC}$ electron bunch at the end of $10 \mathrm{MeV}$ injector.

\begin{tabular}{|l|c|}
\hline$\varepsilon_{\mathrm{x}}(\mathrm{mm}-\mathrm{mrad})$ & 0.8 \\
\hline$\varepsilon_{\mathrm{z}}(\mathrm{deg}-\mathrm{keV})$ & 5 \\
\hline$\sigma_{\mathrm{z}}(\mathrm{ps})$ & 0.5 \\
\hline
\end{tabular}

\subsection{Medium Bunch Charge: $100-300$ pC}

Many high power FEL projects require a bunch charge falling in this range, including Jefferson Lab's UV FEL upgrade plan. There are also 100 to $1000 \mathrm{~kW}$ class IR FEL projects for industrial and other uses being discussed. An improvement of the injector performance over the existing Jlab IR FEL injector is quite impressive. Compare the performance numbers listed in the column under the $135 \mathrm{pC}$ bunch charge case in Tables 1 and 3.

Table 3: Representative beam quality at the end of injector for two different bunch charges.

\begin{tabular}{|l|c|c|}
\hline Bunch Charge & $\mathbf{1 3 5} \mathbf{~ p C}$ & $\mathbf{2 7 0} \mathbf{~ P C}$ \\
\hline$\varepsilon_{\mathrm{x}}(\mathrm{mm}-\mathrm{mrad})$ & 1.6 & 3.5 \\
\hline$\varepsilon_{\mathrm{z}}(\mathrm{deg}-\mathrm{keV})$ & 15 & 26 \\
\hline$\sigma_{\mathrm{z}}(\mathrm{ps})$ & 1.5 & 2 \\
\hline
\end{tabular}

In designing this injector we have not thoroughly searched all the possible optimization routes available. Therefore, we should expect a further improvement on the injector performance by the time a specific project is attached to the design work.

\subsection{High Bunch Charge: $1-2 n C$}

This is the bunch charge range for projects requiring a very high peak current (about $\sim 1 \mathrm{kA}$ ) and/or high average current. For instance, electron ion collider (EIC) projects currently being discussed at several institutes fall into this category. A dc gun based injector is the clearly favored here because of its high $\mathrm{cw}$ beam power necessary for a large luminosity and efficient polarization production capability far superior to any other alternative source. Another example is high power cw FEL (a mega Watt and/or beyond).

Again, we have found an injector solution based on a $500 \mathrm{kV}$ dc gun which can transport 1 2 $\mathrm{nC}$ bunches producing an excellent beam quality. It is worth to point out here that the buncher cavity may need to be replaced by a superconducting one in this example, if a cw operation is desired. Also, beam transport at an injection chicane should be carried out very carefully due to coherent space charge effects in bends. The problem requires a further study. Of course this is a common problem for transporting a high charged bunch whether it is originated from a dc or an rf gun. At moment we should be satisfied with an excellent beam quality we obtain at a $10 \mathrm{MeV}$ point after the acceleration in the cryounit.

Table 4: Injector performance at a $10 \mathrm{MeV}$ location but before an injection chicane which brings the beam to the main linac.

\begin{tabular}{|l|c|c|}
\hline Bunch Charge & 1 nC & 2nC \\
\hline$\varepsilon_{\mathrm{x}}(\mathrm{mm}-\mathrm{mrad})$ & 7.5 & 10 \\
\hline$\varepsilon_{\mathrm{z}}(\mathrm{deg}-\mathrm{keV})$ & 30 & 50 \\
\hline$\sigma_{\mathrm{z}}(\mathrm{ps})$ & 4 & 5 \\
\hline
\end{tabular}

\section{CONCLUSIONS}

The Jlab IR FEL has successfully demonstrated a reliable cw operation of a photocathode dc gun at $5 \mathrm{~mA}$ average current. Experience and research at Jlab have shown that the type of a $500 \mathrm{kV}$ gun assumed for the work reported in this paper should also be available in a not too distant future.

Present work has shown how to properly design a high brightness compact injector built on a photocathode $\mathrm{dc}$ gun and the super-conducting rf cavity. The injector is perfectly suitable for machine projects requiring a high average beam power. Furthermore, the performance of the injector matches with that of the best photocathode rf gun based injector design in many cases[8].

Therefore, we think that a dc gun-based injector is actually the preferred choice for many ongoing accelerator projects which demand a high brightness short bunched beam, if one takes into account its proven operational stability and high average power capability. The Jlab IR FEL Demo holds a world record in average laser beam power mainly due to such a feature in its injector. The ability to produce an electron polarization with a high efficiency is an added advantage.

\section{REFERENCES}

[1] G. R. Neil et al., "Sustained Kilowatt Lasing in a FreeElectron Laser with Same-Cell Energy Recovery", Phys. Rev. Lett., 84, 662 (2000).

[2] B. C. Yunn, "A New Low Emittance Injector for Future FEL Upgrades", Jlab Tech Note, Jlab-TN-01009 (2001).

[3] B. Carsten, "New Photoelectric Injector Design for the Los Alamos National Laboratory XUV FEL Accelerator", Proceedings of the $10^{\text {th }}$ International FEL Conf., Jerusalem (1988).

[4] B. C. Yunn, "An Injector for ERL Project", Jlab Tech Note, Jlab-TN-01-010 (2001).

[5] B. C. Yunn, unpublished work.

[6] PARMELA originated from a code developed by K. Crandall, Los Alamos National Laboratory (1980).

[7] Cornell ERL Report (2001).

[8] e.g. S. G. Biedron, et al., "The Operation of BNL/ATF Gun-IV Photocathode RF Gun at the Advanced Photon Source", Proc. of the 1999 Particle Accelerator Conf. (1999). 